\title{
Simulation Based Sensitivity Analysis of Split Ring Resonator for Detecting Compositional Variations in Mixture of Polar Liquids
}

\author{
M. Haris Amir, Muhammad Naveed, Tahir Ejaz, Tahir Zaidi, Muhammad Shoaib, Mashhood Ahmad
}

\begin{abstract}
Split ring resonator is area of major research in recent years especially in the field of fluid sensing, because of their ease in fabrication and moderate quality factor and resonant frequencies. Split ring resonator provide shift in resonant frequency after system is perturbed by introducing a small amount of fluid into the system. In this paper a simulation based sensitivity analysis has been carried out to choose a split ring resonator which can provide maximum shift in resonant frequency when a sample mixture of polar liquids perturbs the system. Simulation results show effect of length of split ring resonator and orientation of micro-capillary tube on resonant frequency and quality factor. A comparison has been presented on obtained results.
\end{abstract}

Keywords---Split ring resonator, resonator length, micro-capillary tube, orientation, perturbation

\section{Introduction}

Study of compositional and molecular structure of various polar liquids is very useful for synthesizing process in pharmaceutical, chemical, fertilizer, food processing and other industries. Permittivity is used to find knowledge regarding compositional and molecular structure of materials and material behavior in electromagnetic environment [1] [2] [3]. There are two major microwave methods to explore composition of polar liquids i.e., non-resonant methods and resonant

Authors Names / Affiliation M. Haris Amir National University of Sciences and Technology, Pakistan.

Tahir Ejaz

HITEC University, Pakistan

Muhammad Shoaib

National University of Sciences and Technology, Pakistan
Muhammad Naveed

National University of Sciences and Technology, Pakistan.

Tahir Zaidi

National University of Sciences and Technology, Pakistan.

Mashhood Ahmad

National University of Sciences and Technology, Pakistan methods. Resonant method provides solution

at discrete frequencies [4] and considered more precise as compared to non-resonant methods which provide solution at broadband frequencies [5].

Non-resonant methods are categorized amongst reflection and transmission methods and includes coaxial probe, parallel plate measurements and open space systems [6]. Resonant method on the other hand includes perturbation technique for which resonators of different configuration are utilized.

In resonant perturbation method, material under test (MUT) is introduced inside the gap of split ring resonator (SRR), as shown in Figure 1. Introduction of MUT changes the electromagnetic properties of the system [7]. These changes can be measured in form of shifts in resonant frequency $f_{r}$ and quality factor $Q[8]$.

In this paper, sensitivity analysis of SRR with micro-capillary tube in different orientations have been discussed along with effects of resonator length.

\section{Theory}

SRR is a lumped-element device that can be modeled using $L C$ circuit. A simple SRR structure is shown in Figure 1. Metallic cylindrical loop acts as inductor while longitudinal gap acts as a capacitor [9]. Magnetic field (H-field) surround the SRR's loop whereas electric field (E-field) is induced inside the resonator gap [10] . Mathematical relation for
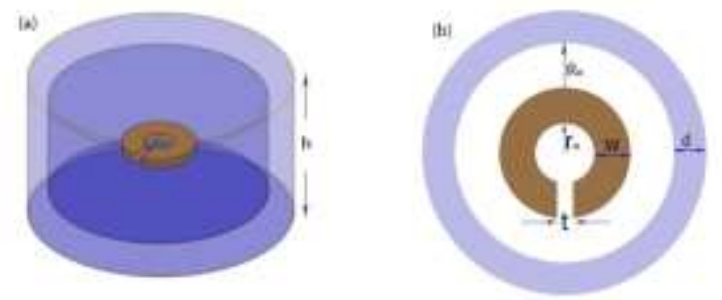

Figure 1 Structure for split ring resonator (a) Design of split ring resonator in metallic shield cavity (b) cross sectional view of split ring resonator with shield cavity

resonant frequency of $L C$ circuit is 
Proc. of the Seventh International Conference On Advances in Computing, Electronics and Electrical Technology - CEET 2017. Copyright (C) Institute of Research Engineers and Doctors. All rights reserved.

ISBN: 978-1-63248-126-9 doi: 10.15224/ 978-1-63248-126-9-29

$$
f_{r}=\frac{1}{2 \pi \sqrt{L C}}
$$

In above equation $L$ represents the overall inductance while $C$ represents overall capacitance. Similarly mathematical relation for quality factor of $L C$ circuit is

$$
Q=\frac{\omega}{\Delta \omega}=\frac{f_{r}}{\Delta f_{r}}
$$

Perturbation is achieved when small amount of dielectric samples, with some value of relative permittivity, is placed in resonant structure. Due to which both the above mentioned resonant parameters changes. Relation between real and imaginary parts of permittivity, $f_{r}$ and $Q$ was observed as [11]

$$
\begin{aligned}
\varepsilon_{r}^{\prime} & =\Delta f_{r} \\
\varepsilon_{r}^{\prime \prime} & =\Delta\left(\frac{1}{Q}\right)
\end{aligned}
$$

SRR is enclosed inside a metallic cavity to prevent excessive radiation loss, thereby maintaining high $Q$ for microwave frequencies. Micro-capillary tube containing solvents can be introduced either parallel to E-field or perpendicular to E-field inside the capacitive split [9] which in turn perturbs properties of system [12]. Both orientations of microcapillary tube are discussed in detail in this paper.

\section{Design Structure and Simulation}

Ansoft HFSS (version 13) [13] was used to design 3-D model of SRR with micro-capillary tube in different orientations. These models were divided into two sub categories.

Category 1: Micro-capillary tube introduced into SRR gap horizontal to E-field

Category 2: Micro-capillary tube introduced in SRR gap vertical to E-field.

Optimized parameters of SRR and cylindrical cavity worked out in earlier research [14] is shown in Table 1. Aluminum and copper was used to design cylindrical cavity and SRR respectively [15]. Simulations pertaining to both categories are discussed below.

Category 1: This design involves a SRR, enclosed inside a shield, with a micro-capillary tube placed in horizontal direction of E-field. To sense frequency shift pertaining to polar liquids, mixture of ethanol and methanol was used in different composition inside the micro-capillary tube at room temperature $\left(25^{0} \mathrm{C}\right)$ in Ansoft HFSS. Micro-capillary tube containing sample liquid mixture was introduced horizontally from the side wall of shield cavity and inside the SRR gap. HFSS parametric setup was designed for sample liquid within micro-capillary tube. Range for real and imaginary parts of relative permittivity for the two liquids for parametric setup is given in Table 2 [16]. Another parametric setup for length of resonator from $3 \mathrm{~mm}$ to $15 \mathrm{~mm}$ with the step size of $3 \mathrm{~mm}$ was also designed in Ansoft HFSS. Results of different values for permittivity at different resonator lengths is shown in Figure 2.

Category 2: This design is similar to category 1 except for the orientation of micro-capillary tube which was placed vertical to E-field i.e., microcapillary tube was introduced vertically from the top of shield cavity inside the SRR gap. Same parametric setups were designed as in category 1 . Results were obtained which are shown in Figure 3.

TABLE 1 - OPTIMIZED DESIGN PARAMETERS

\begin{tabular}{lc}
\hline Design Parameters & Values \\
\hline Inner Radius of Outer Shield ' $\mathrm{R}_{0}$ ' (mm) & 22 \\
Inner Radius of SRR ' $\mathrm{r}_{0}$ ' (mm) & 5 \\
Width of SRR 'W' (mm) & 6 \\
Gap of SRR ' $\mathrm{t}$ ' (mm) & 2.2 \\
Height of Shield 'h' (mm) & 32 \\
Thickness of Outer Shield 'd' (mm) & 7
\end{tabular}

TABLE 2 - REAL AND IMAGINARY PART OF RELATIVE PERMITIVITY OF ETHANOL AND METHANOL AT $0.1 \mathrm{GHz} \& 25^{\circ} \mathrm{C}$

Ethanol Methanol
$\varepsilon_{r}^{\prime}=24.43$
$\varepsilon_{r}^{\prime}=32.66$
$\varepsilon_{r}^{\prime \prime}=2.05$
$\varepsilon_{r}^{\prime \prime}=0.86$ 
Proc. of the Seventh International Conference On Advances in Computing, Electronics and Electrical Technology - CEET 2017. Copyright $(\subseteq$ Institute of Research Engineers and Doctors. All rights reserved.

ISBN: 978-1-63248-126-9 doi: 10.15224/ 978-1-63248-126-9-29

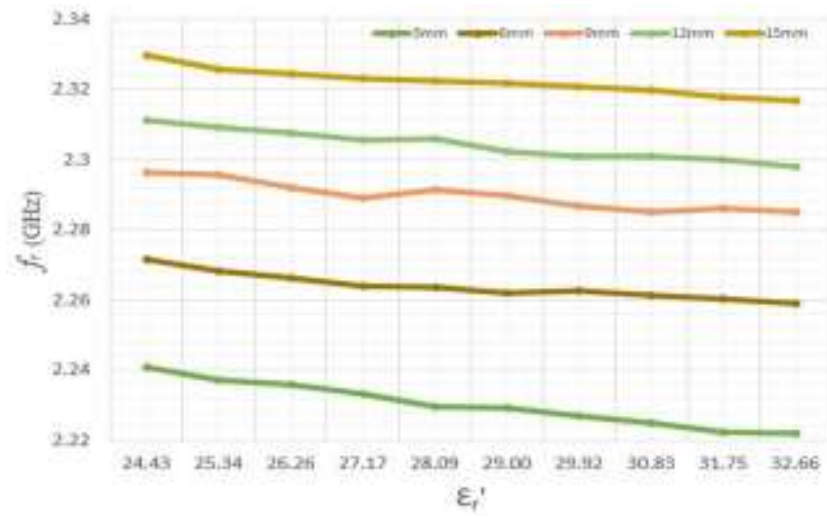

(a) Resonant frequency as a function of real part of permittivity

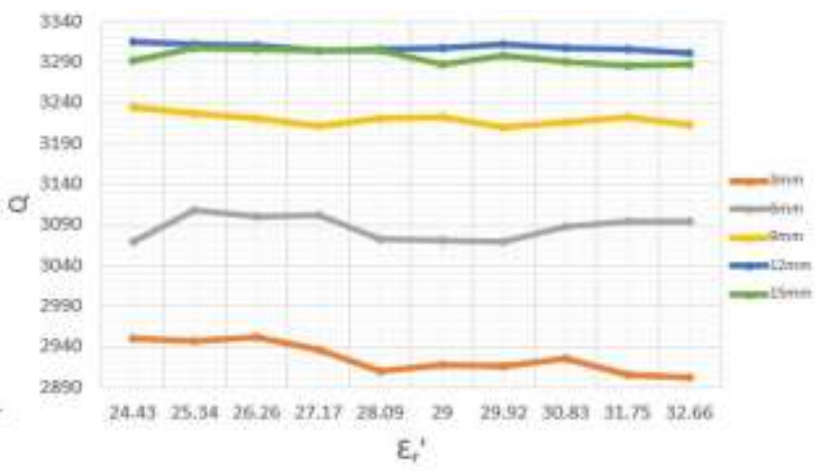

(b) Quality factor as a function of real part of permittivity

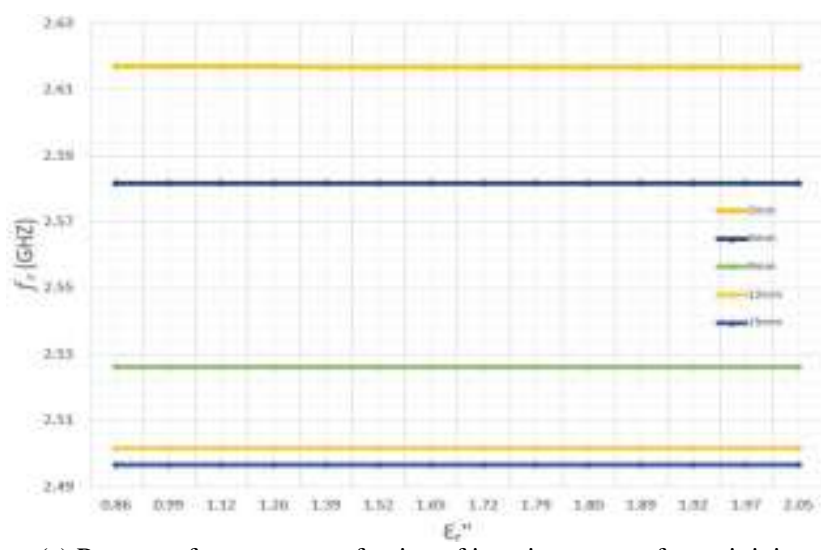

(c) Resonant frequency as a funtion of imaginary part of permittivity

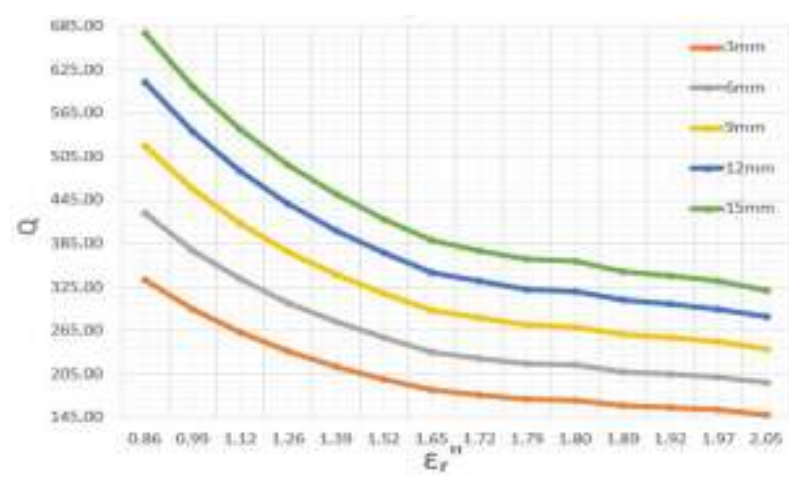

(d) Quality factor as a funtion of imaginary part of permittivity

Figure 2. Resonator parameters for horizontally oriented micro-capillary tube

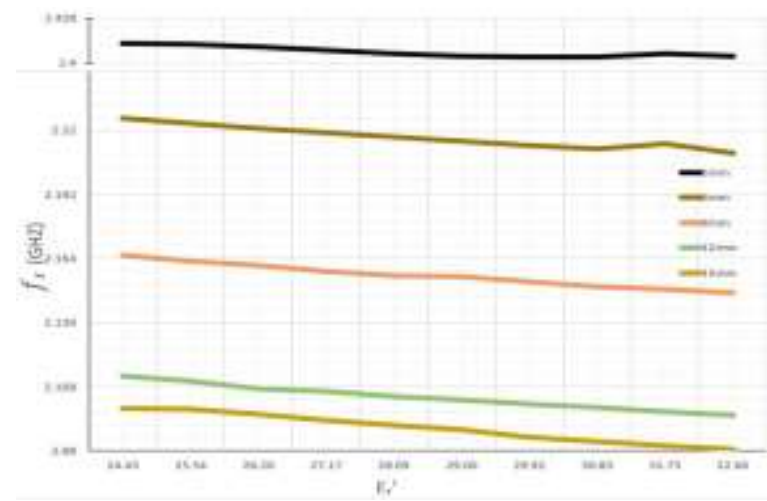

(a) Resonant frequency as a function of real part of permittivity

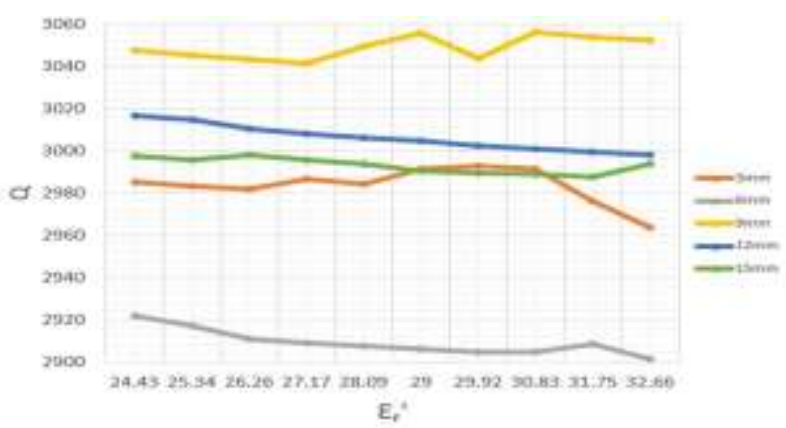

(b) Quality factor as a function of real part of permittivity

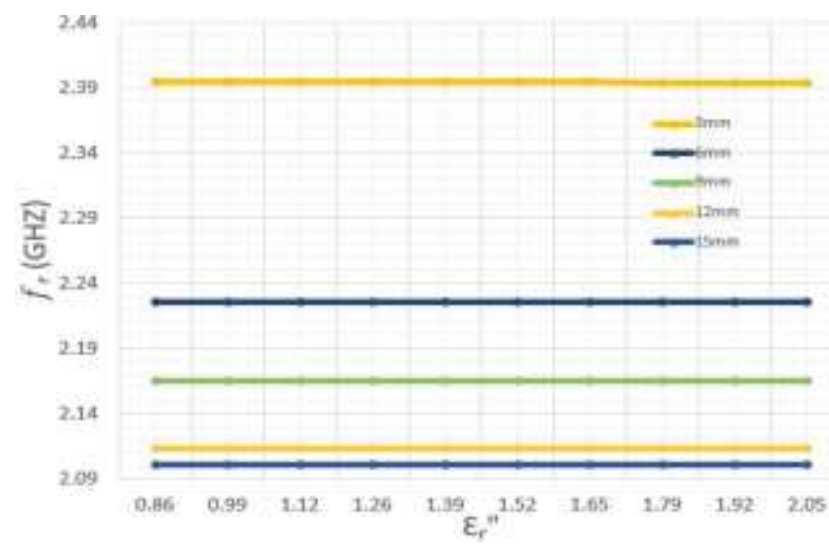

(c) Resonant frequency as a function of imaginary part of permittivity

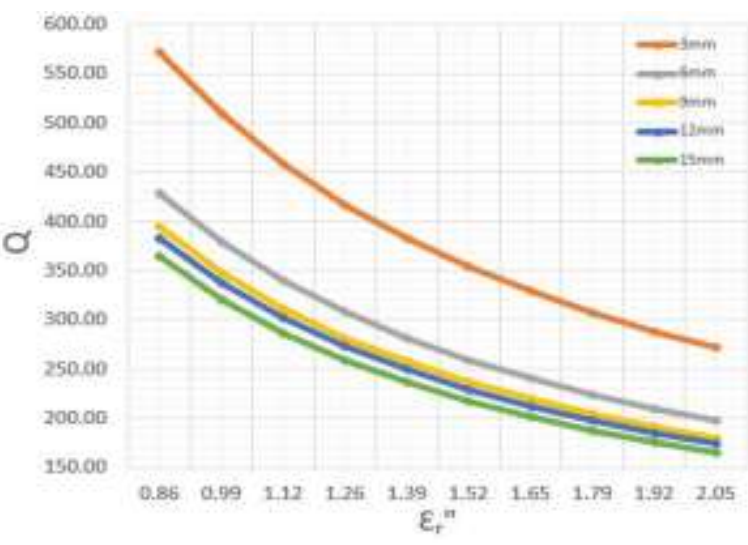

(d) Quality factor as a function of imaginary part of permittivity

Figure 3. Resonator parameters for vertically oriented micro-capillary tube 


\section{Analysis and Discussion}

In this section simulation results of two different configurations of SRRs have been discussed, analyzed and compared with respect to the shift in $f_{r}$ and change in $Q$.

Category 1: Figure 2 shows the response of system when real and imaginary part of MUT was varied depicting change in liquid composition. It can be observed from Figure 2, that resonance frequency $f_{r}$ of system varies when real part of permittivity changes. Whereas $Q$ of the system varies when imaginary part of permittivity changes. It can also be observed from Figure 2(a) that the maximum shift in $f_{r}$ occurs when resonator height is $3 \mathrm{~mm}$.

Category 2: Figure 3 shows response of system when real and imaginary part of permittivity of MUT is varied. Similar kind of results were achieved in this configuration also. It was observed that in this case, maximum shift in $f_{r}$ was achieved for resonator length of $15 \mathrm{~mm}$.

Detailed results of shift in $f_{r}$ for both categories is shown in Table 3. It can also be observed that for vertical orientation of micro-capillary tube, frequency shift increases with increase in SRR length.

Comparison: Figure 4 shows comparison between category 1 and category 2 . It can be observed that placement of micro-capillary tube both in horizontal and vertical orientations yielded almost same frequency shift but at different resonator length. Figure 4(a) shows the variation in $f_{r}$ with respect to the real part of permittivity in both configurations. It can be observed that for horizontally orientated micro-capillary tube, frequency shift for the given range of real permittivity is $19 \mathrm{MHz}$ at resonator height of $3 \mathrm{~mm}$. Whereas in case of vertically oriented micro-capillary tube, frequency shift is also $19 \mathrm{MHz}$ but at resonator height of $15 \mathrm{~mm}$ as shown in Table 3. As against equation (3) and (4) mentioned above, slight change in $Q$ was noted for the variation in real part of permittivity which is shown in Figure 4(b) which shows that real part of permittivity has a negligible effect on $\mathrm{Q}$ which can be ignored. Figure 4(c) shows marginal shift in $f_{r}$ with respect to variation in imaginary part of permittivity while Figure 4(d) shows continuous variation in $Q$ with change in imaginary part of permittivity. It can also be observed that $Q$ changes very smoothly when imaginary part of permittivity changes. Frequency shift for each resonator length in both orientations is given in Table 3.

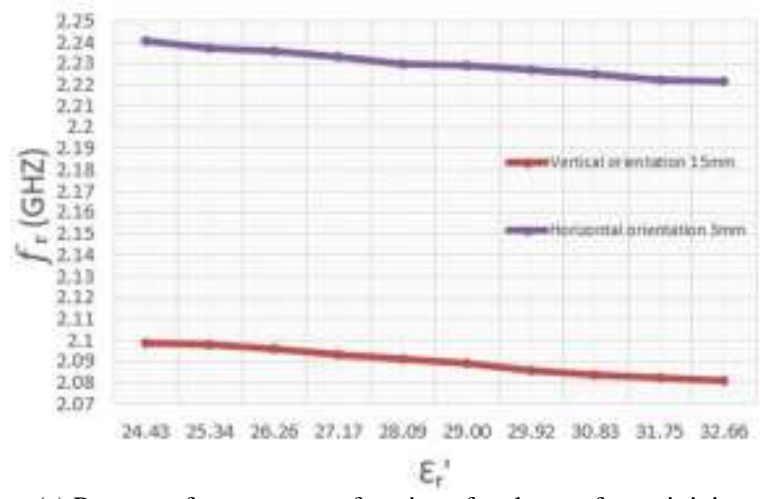

(a) Resonant frequency as a function of real part of permittivity

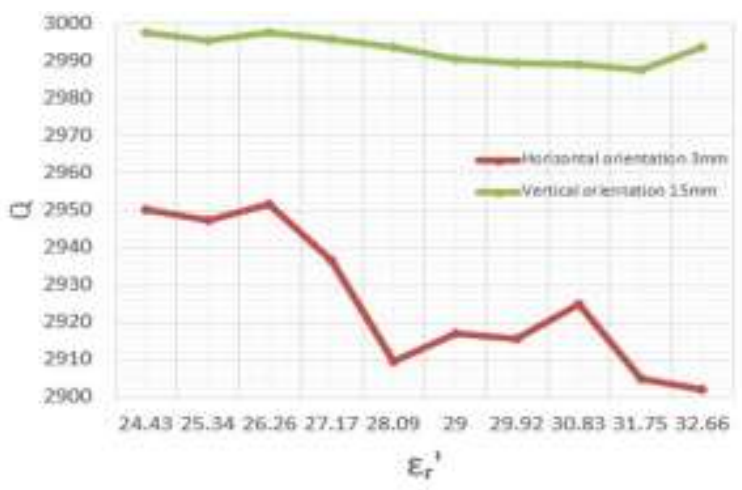

(b) Quality factor as a function of in real part of permittivity

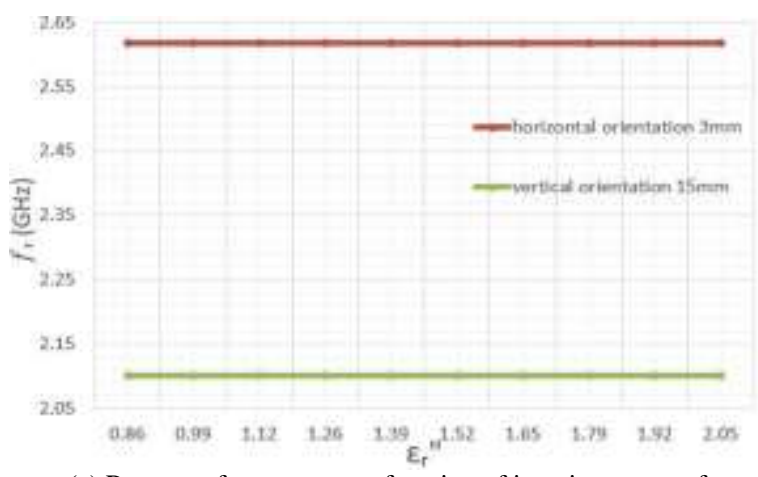

(c) Resonant frequency as a function of imaginary part of permittivity

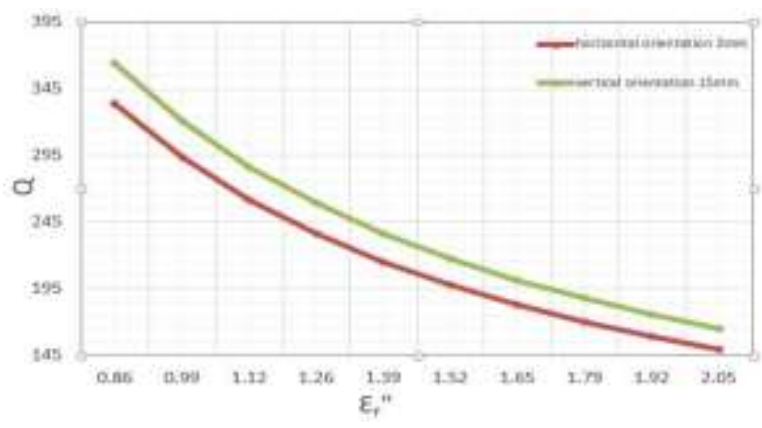

(d) Quality factor as a function of imaginary part of permittivity

Figure 4. Comparison between resonator parameters for both orientations 
Proc. of the Seventh International Conference On Advances in Computing, Electronics and Electrical Technology - CEET 2017. Copyright (C) Institute of Research Engineers and Doctors. All rights reserved.

ISBN: 978-1-63248-126-9 doi: 10.15224/ 978-1-63248-126-9-29

TABLE 3 - FREQUENCY SHIFT FOR EACH SIMULATION

\begin{tabular}{|c|c|c|c|c|c|c|}
\hline \multirow{2}{*}{$\begin{array}{c}\text { Resonator } \\
\text { Length } \\
(\mathbf{m m})\end{array}$} & \multicolumn{3}{|c|}{ Horizontally oriented micro-capillary tube } & \multicolumn{3}{|c|}{ Vertically oriented micro-capillary tube } \\
\cline { 2 - 7 } & $\begin{array}{c}\operatorname{Max} f_{r} \\
(\mathrm{GHz})\end{array}$ & $\begin{array}{c}\operatorname{Min} f_{r} \\
(\mathrm{GHz})\end{array}$ & $\begin{array}{c}\Delta f_{r} \\
(\mathrm{GHz})\end{array}$ & $\begin{array}{l}\text { Max } f_{r} \\
(\mathrm{GHz})\end{array}$ & $\begin{array}{c}\text { Min } f_{r} \\
(\mathrm{GHz})\end{array}$ & $\begin{array}{c}\Delta f_{r} \\
(\mathrm{GHz})\end{array}$ \\
\hline 3 & 2.241 & 2.222 & $0.019=19 \mathrm{MHz}$ & 2.412 & 2.404 & $0.008=8 \mathrm{MHz}$ \\
\hline 6 & 2.272 & 2.259 & $0.013=13 \mathrm{MHz}$ & 2.225 & 2.210 & $0.015=15 \mathrm{MHz}$ \\
\hline 9 & 2.297 & 2.285 & $0.011=11 \mathrm{MHz}$ & 2.165 & 2.149 & $0.016=16 \mathrm{MHz}$ \\
\hline 12 & 2.311 & 2.297 & $0.013=13 \mathrm{MHz}$ & 2.113 & 2.094 & $0.018=18 \mathrm{MHz}$ \\
\hline 15 & 2.330 & 2.317 & $0.013=13 \mathrm{MHz}$ & 2.099 & 2.080 & $0.019=19 \mathrm{MHz}$ \\
\hline
\end{tabular}

\section{Conclusion}

In this paper a simulation based sensitivity analysis of different lengths of SRR for vertical and horizontal orientation of micro-capillary tube has been presented to obtain maximum shift in resonant frequency. Simulations results shows that both vertical and horizontal orientation of micro-capillary tube provide approximately same shift in resonant frequency but at different resonator lengths. However, vertically oriented micro-capillary tube provides better results as compared to horizontally oriented micro-capillary tube because its frequency shift improves with the increase in the SRR's length.

Work produced in this paper provides guidelines for designing resonator with MUT placed in both orientations. Sensitivity analysis is a key to choose amongst the two orientations.

\section{References}

[1] M. Sharma, R. Resta, and R. Car, "Dipolar Correlations and the Dielectric Permittivity of Water," Phys. Rev. Lett., vol. 98, no. 24, p. 247401, Jun. 2007.

[2] M. Halik et al., "Relationship Between Molecular Structure and Electrical Performance of Oligothiophene Organic Thin Film Transistors," Adv. Mater., vol. 15, no. 11, pp. 917-922, Jun. 2003.

[3] S. O. Nelson, "Dielectric properties of agricultural productsmeasurements and applications," IEEE Trans. Electr. Insul., vol. 26, no. 5, pp. 845-869, Oct. 1991.

[4] D. J. Rowe, S. al-Malki, A. A. Abduljabar, A. Porch, D. A. Barrow, and C. J. Allender, "Improved Split-Ring Resonator for Microfluidic Sensing," IEEE Trans. Microw. Theory Tech., vol. 62, no. 3, pp. 689-699, Mar. 2014.

[5] A. Schönhals and F. Kremer, "Broadband Dielectric Measurement Techniques (10-6 Hz to $1012 \mathrm{~Hz}$ )," in Broadband Dielectric Spectroscopy, P. D. F. Kremer and P.D. D. A. Schönhals, Eds. Springer Berlin Heidelberg, 2003, pp. $35-57$.

[6] O. V. Tereshchenko, F. J. K. Buesink, and F. B. J. Leferink, "An overview of the techniques for measuring the dielectric properties of materials," in General Assembly and Scientific Symposium, 2011 XXXth URSI, 2011, pp. 1-4.

[7] J. A. Pereda, L. A. Vielva, A. Vegas, and A. Prieto, "Computation of resonant frequencies and quality factors of open dielectric resonators by a combination of the finite- difference time-domain(FDTD) and Prony's methods," IEEE Microw. Guid. Wave Lett., vol. 2, no. 11, pp. 431-433, 1992.

[8] S.-Y. Cho, I.-T. Kim, and K. S. Hong, "Microwave dielectric properties and applications of rare-earth aluminates," $J$. Mater. Res., vol. 14, no. 1, pp. 114-119, Jan. 1999.

[9] Adnan Masood, Adrian Porch, and David Barrow, "Microwave Resonators for Highly Sensitive Compositional Analysis: Applications for Solvents in Microcapillary Systems," Phd, Cardiff University.

[10] M. Mehdizadeh and T. K. Ishii, "Electromagnetic field analysis and calculation of the resonance characteristics of the loop-gap resonator," IEEE Trans. Microw. Theory Tech., vol. 37, no. 7, pp. 1113-1118, Jul. 1989.

[11] T. Hayat, T. Ejaz, M. Naveed, T. Zaidi, and H. U. Rahman, "Simulation Based Comparative Analysis of Split Resonators for Compositional Analysis," in The Fourth International Conference on Technological Advances in Electrical, Electronics and Computer Engineering (TAEECE2016), 2016, p. 29.

[12] L.-F. Chen, C. K. Ong, C. P. Neo, V. V. Varadan, and V. K. Varadan, Microwave electronics: measurement and materials characterization. John Wiley \& Sons, 2004.

[13] "ANSYS HFSS: High Frequency Electromagnetic Field Simulation." [Online]. Available: http://www.ansys.com/products/\%20electronics/ansys-hfss. [Accessed: 24-Apr-2017].

[14] T. Ejaz, H. U. Rahman, S. A. A. Shah, and T. Zaidi, "A comparative analysis of split-ring resonator models," in Proceedings of the 4th International Conference on Informatics, Electronics \& Vision (ICIEV'15), 2015, p. 61.

[15] T. Ejaz, H. Ur Rahman, T. Tauqeer, A. Masood, and T. Zaidi, "Shield optimization and formulation of regression equations for split-ring resonator," Math. Probl. Eng., vol. 2016, 2016.

[16] A. P. Gregory and R. N. Clarke, Tables of the complex permittivity of dielectric reference liquids at frequencies up to $5 \mathrm{GHz}$. National Physical Laboratory Teddington, 2001. 
Proc. of the Seventh International Conference On Advances in Computing, Electronics and Electrical Technology - CEET 2017.

Copyright (C) Institute of Research Engineers and Doctors. All rights reserved.

ISBN: 978-1-63248-126-9 doi: 10.15224/ 978-1-63248-126-9-29

\section{About Authors}

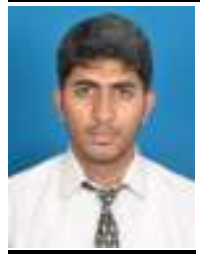

M. Haris Amir received the B.Sc degree in Electrical Engineering from Air University, Islamabad, Pakistan in 2014. Presently he is working toward the Master degree in Microwave Engineering at College of Electrical and Mechanical Engineering, National University of Sciences and Technology, Islamabad, Pakistan. His current area of research is microwave resonators.

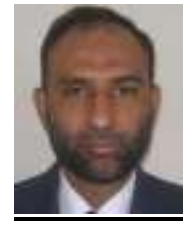

Muhammad Naveed received his $\mathrm{Ph} . \mathrm{D}$. degree in high frequency electromagnetics from Quaid-i-Azam University, Islamabad, Pakistan. Currently, he is Associate Professor with department of Electrical Engineering, College of Electrical and Mechanical Engineering, National University of Sciences and Technology, Islamabad, Pakistan.

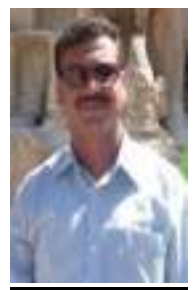

Tahir Ejaz received his B.Sc degree in Electrical Engineering from University of Engineering and Technology, Lahore, Pakistan and M.Sc. degree in Electrical Engineering from College of Electrical and Mechanical Engineering, National University of Sciences and Technology, Islamabad, Pakistan in Electrical Engineering. Currently, he is Assistant Professor with Department of Electrical Engineering, HITEC University, Taxila, Pakistan. His current research are is material characterization using microwave techniques.

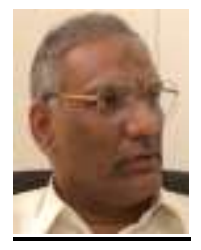

Tahir Zaidi received his $\mathrm{Ph} . \mathrm{D}$. degree from Georgia Institute of Technology, School of Electrical and Computer Engineering, United States in 2010 in Compound Semiconductors. Currently, he is Associate professor with Department of Electrical Engineering, College of Electrical and Mechanical Engineering, National University of Sciences and Technology, Islamabad, Pakistan.

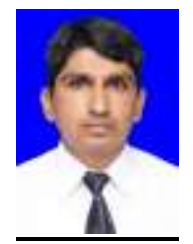

Muhammad Shoaib received his B.Sc degree in Electrical Engineering from College of Electrical and Mechanical Engineering, National University of Sciences and Technology, Islamabad, Pakistan. Presently, he is working toward Master degree in Microwave Engineering. His research concerns are design of tunable split ring resonators.

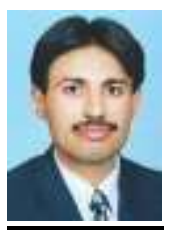

Mashhood Ahmad received his Ph.D. degree from Quaid-i-Azam University, Islamabad in quantum optics. He worked on quantum state measurement schemes and entanglement dynamics. Currently, he is in the faculty of Department of Electrical Engineering, College of Electrical Engineering, National University of Sciences and Technology, Islamabad, Pakistan. 